\title{
Salud mental, habilidades de afrontamiento y rendimiento académico en estudiantes universitarios
}

\author{
Mental health, coping skills and academic performance \\ in undergraduate students
}

Nehemías Cuamba Osorio

Nitzia Astrid Zazueta Sánchez

Universidad de Sonora

\section{Resumen}

El ambiente universitario es un contexto que ofrece retos a los estudiantes y probabiliza la presencia de problemas de salud mental si estos no cuentan con habilidades necesarias para hacerles frente, fenómeno que afecta diversas áreas. Partiendo de los objetivos, identificar la presencia de indicadores de problemas de salud mental y habilidades de afrontamiento en los estudiantes universitarios, y analizar las diferencias entre las variables relacionadas a problemas de salud mental y habilidades de afrontamiento entre grupos de rendimiento académico en estudiantes universitarios, se estructuró una investigación cuantitativa y transversal, con una muestra de 688 estudiantes, a los cuales se les aplicó dos instrumentos, uno de salud mental y otro de habilidades de afrontamiento; se analizaron los resultados a nivel global y por carrera, además de buscar si presentaban diferencias con el rendimiento académico. Los resultados demostraron que más de la mitad de los estudiantes presentó problemas de salud mental, así como problemas en la ejecución de habilidades de afrontamiento; no hubo diferencias significativas en el análisis comparativo, se discuten los resultados.

Palabras clave: salud mental, rendimiento académico, habilidades de afrontamiento, estudiantes universitarios

\footnotetext{
Nota del autor

Nehemías Cuamba Osorio, Departamento de Psicología, Universidad de Sonora. Nitzia Astrid Zazueta Sánchez Departamento de Psicología, Universidad de Sonora. La correspondencia en relación con este artículo debe dirigirse a Nehemías Cuamba Osorio, Universidad de Sonora, Av Unison No. 343, 84000 Nogales, Son.

Dirección electrónica: nehemias.cuamba@unison.mx
}

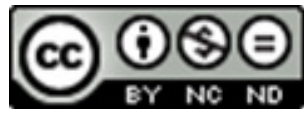




\begin{abstract}
The university environment is a context that presents challenges to the student and increases the likelihood of mental health problems if they do not possess the skills required to face them, a phenomenon that affects different spheres. Aiming to identify the presence of mental health problems indicators and coping skills in undergraduate students, and to analyze the differences between the variables related to mental health problems and coping skills between groups of academic performance in undergraduate students, a quantitative cross-sectional research was structured. A sample of 688 answered two instruments, one to evaluate mental health and another one to assess coping skills. Results were analyzed globally and by career, and differences in academic performance were searched. Results showed that more than half of the students presents indicators of mental health problems, as well as problems in the execution of coping skills. There were no differences in the comparative analysis, results are discussed.
\end{abstract}

Keywords: Mental health, academic performance, coping skills, undergraduate students

El estudio de la salud mental (en cualquier contexto) es complejo, dado que en el constructo convergen una gama de factores de índole biológico, psicológico, social y económico (Organización Mundial de la Salud, 2018), factores que interactúan y dependen entre sí, que probabilizan el desarrollo de un estado de bienestar donde la persona es consciente de sus herramientas para hacer frente a situaciones estresantes de la vida, lo cual le permite funcionar socialmente (Souza y Cruz, 2010). A su vez, Restrepo y Jaramillo (2012), según el modelo cognitivo, refieren que la salud mental es la capacidad de adaptación del individuo ante las demandas del ambiente. Las afirmaciones anteriores denotan que el concepto ya no hace alusión solamente a la ausencia de trastornos mentales, sino que integra indicadores de bienestar y afrontamiento en el individuo.
Según el Modelo General de Salud Mental propuesto por Barrón y Sánchez (2001), estos indicadores de bienestar incluyen dos niveles de mediación. El primero es el ambiental y social, que engloba el estrés, la alienación y la integración comunitaria. El segundo es el psicosocial, que engloba el apoyo social y factores psicológicos como autoestima y estilos de afrontamiento; estos probabilizan la presencia o ausencia de lo que los autores llaman deterioro psicológico.

Bajo esta perspectiva, los recursos mencionados son conocidos como habilidades de afrontamiento, concepto acuñado en el Modelo Transaccional del Estrés por Lazarus y Folkman (1986). Estos autores plantearon que estas habilidades son aquellas estrategias dirigidas a resolver demandas evaluadas por la persona como desbordantes de sus propios 
recursos, haciendo mención de que las estrategias se dividían en dos: 1) aquellas centradas en el problema, las cuales tienen relación sobre el estresor con el objetivo de reducir su impacto; y 2) aquellas centradas en la emoción (previene, minimiza y reduce el estrés).

Conforme se fue estudiando el constructo, se determinó que estas habilidades también podían dividirse en activas (que corresponde a un manejo adecuado centrado tanto en el problema como la emoción, con el objetivo de lograr el control de emociones, reflexionar posibles soluciones, anticipación al desastre y búsqueda de apoyo social) y pasivas (manejo inadecuado centrado en el problema y la emoción que orienta hacia la culpabilidad, el rechazo y el retraimiento social con la esperanza que el problema se resuelva por sí solo) (Sepúlveda et al., 2012). Según Cassaretto (2010) tanto las estrategias centradas en el problema como las activas son importantes para la adaptación del individuo y son determinantes para que la persona tenga un nivel adecuado de bienestar y salud mental.

Sin embargo, la OMS (2019) señala que los problemas de salud mental van en aumento, refiriendo que la prevalencia de trastornos mentales se encuentra al alza, dado que la prospección en prevalencia está cerca del $20 \%$ de la población mundial y sus efectos generan consecuencias socioeconómicas a nivel mundial. La OMS también menciona que en países en vías de desarrollo 76 a $85 \%$ de la población no recibe atención adecuada. En México, 1 de cada
4 adultos de 18 a 65 años ha padecido o padece algún tipo de trastorno mental (Secretaría de Salud, 2013); si tomamos en cuenta lo referido por la OMS, de los cuatro mencionados solo 1 o menos recibe o recibirá tratamiento adecuado, además investigaciones refieren que la población con esta clase de problemas tarda entre 4 a 20 años en buscar algún tipo de apoyo (Berenzon et al., 2013), por lo cual el problema se vuelve mayor.

Dado el rango de población, este tipo de problemas de salud mental se ve reflejado en el entorno universitario. El contexto universitario es un ambiente en donde el alumnado se ve expuesto a exigencias y demandas, dado que en este periodo convergen factores como los trabajos escolares, sobrecarga académica, participaciones en clase, entre otras obligaciones académicas (García et al., 2012; Pozos et al., 2015), con la etapa evolutiva, adquisición de mayor autonomía, y la competitividad ante las expectativas personales, sociales y familiares (Antuñez y Vinet, 2013; Badder et al. 2014).

Si a lo anterior, se suma un déficit en habilidades de afrontamiento a problemas de la vida académica y actividades externas (Prado et al., 2015), se probabiliza la presencia de periodos de estrés que afectan la salud mental y el rendimiento académico (El Ansari et al., 2014; Ferrel et al., 2014; Pérez et al., 2011; Pozos et al., 2014). Con relación a estas variables, Barraza (2006), propuso el modelo sistémico cognoscitivista para el estudio del estrés académico, el cual indica que, si el alumno 
se percibe sometido a demandas escolares, $\mathrm{y}$ estas son valoradas como estresores, provocará un desequilibrio sistémico que se manifiesta a través de problemas de salud mental.

Diversos estudios hacen alusión al fenómeno; Micin y Bagladi (2011) encontraron una alta prevalencia de trastornos mentales en universitarios, siendo los trastornos adaptativos (48.3), los trastornos del estado de ánimo (22.8) y los trastornos ansiosos (20.9) los de mayor presencia. Gallagher (2014) encontró que en Estados Unidos (en una muestra de casi 100 000 estudiantes) un $15.8 \%$ de estudiantes universitarios presentaron trastornos de ansiedad y un $13.1 \%$ trastornos depresivos, señaló que los problemas de salud mental han aumentado en los últimos cinco años en los jóvenes universitarios estadounidenses.

Chau y Vilela (2017) en un estudio con 520 estudiantes universitarios encontraron que los alumnos con menor dificultad académica presentaron mejores condiciones en salud mental, y un menor nivel de estrés. Entre los desórdenes prevalentes en muestras tomadas a este nivel educativo se encuentran, además de los anterior mencionados, conductas de autodestrucción como lo son los comportamientos suicidas (Lester, 2014; Lipson et al., 2015); asimismo, la presencia de una pobre calidad de sueño (Valerio, Jim et al., 2016) y el aumento de conductas adictivas o de consumo de sustancias (Sinha y Jastreboff, 2013).

Con relación a estudios de habilidades de afrontamiento, Marenco et al. (2017), retomaron el término de "Burnout Académico" debido a la exposición prolongada de estresores en el estudiante, lo cual manifiesta su pobre capacidad de afrontamiento y adaptación ante estas situaciones. Byrd y McKinney (2012) encontraron que el afrontamiento es una variable que probabiliza una mejor salud mental en estudiantes universitarios. Pritchard et al. (2007) en su estudio concluyeron que las habilidades de afrontamiento se relacionaban con una adecuada capacidad de adaptación a las presiones $\mathrm{y}$ desafíos del entorno universitario. Lo anterior pone de manifiesto la necesidad de desarrollar habilidades cognitivas de afrontamiento que ayuden al estudiante universitario en su salud mental en vías de mejorar su rendimiento académico (Sánchez et al., 2015).

En México, en especial en ciudades fronterizas donde la población tiende a ser flotante y en constante rotación, se probabiliza una mayor presencia de esta clase de problemas de salud mental, dado que se presenta un alto índice de desintegración en la dinámica familiar (Jurado, 2016). Este es un factor necesario para que el joven desarrolle estrategias de adaptación ante las dificultades que se presentan en la vida diaria y desarrolle las habilidades necesarias para afrontar las situaciones que se perciban como fuera de su control. No obstante, en México se ha subestimado la importancia de estas variables personales asociadas al funcionamiento de la salud mental, sobre todo en cómo se asocia con el rendimiento académico de los estudiantes 
(Caso y Hernández, 2010), por lo cual se pueden encontrar pocos estudios relacionados al tema.

En la Universidad de Sonora (UNISON) específicamente en campus Nogales ha aumentado la presencia de múltiples casos de comportamientos que hacen pensar en la presencia de problemas de salud mental en los estudiantes, como lo son comportamientos parasuicidas (ideación y planeación suicida, así como autolesiones). Además, se han reportado varios sucesos de suicidios consumados, comórbido a eventos donde se presentan en los estudiantes problemas de pánico y angustia e inestabilidad emocional, lo anterior, reportado por diferentes maestros y por la encargada de bienestar psicológico (V. Lara, comunicación personal, 14 de octubre 2019). A su vez, se presenta el alza en la cantidad de población estudiantil, aunado a poco personal tutorial para atención y seguimiento, lo cual ha dificultado llevar un monitoreo con relación a este tipo de problemas en los estudiantes, afectando que se puedan llevar acabo adecuados métodos para prevenir este tipo de situaciones.

Dado lo anterior, el presente estudio parte de las siguientes preguntas de investigación: ¿se presentarán indicadores de problemas de salud mental y problemas en habilidades de afrontamiento en los estudiantes de Universidad de Sonora campus Nogales?, y en caso de presencia ¿cómo se presentan estos problemas de salud mental y habilidades de afrontamiento con relación al rendimiento académico en estudiantes de Universidad de Sonora campus
Nogales? Se parte, a su vez, de los objetivos generales: identificar la presencia de indicadores de problemas de salud mental y habilidades de afrontamiento en los estudiantes universitarios y analizar las diferencias entre las variables relacionadas a problemas de salud mental y habilidades de afrontamiento entre grupos de rendimiento académico en estudiantes universitarios

\section{Método}

\section{Tipo de estudio}

La presente investigación tiene un diseño no experimental u observacional, cuantitativo, transversal de carácter exploratorio y descriptivo.

\section{Participantes}

Para la estructuración de la muestra se utilizó un muestreo no probabilístico compuesto por sujetos voluntarios. De inicio se contempló la aplicación de los instrumentos a toda la población estudiantil del campus, no obstante, como criterio de inclusión se estableció que fueran estudiantes con credencial vigente y que hubiera disposición a participar, por ende no se consideraron criterios de exclusión, como criterio de eliminación que el estudiante no haya contestado en su totalidad los cuestionarios aplicados (esto con la intención de reducir al máximo los valores perdidos). Por ello, de una población de casi 1100 alumnos resultó al final una $\mathrm{n}=688$ estudiantes de la Universidad de Sonora campus Nogales de las 5 carreras, representados en porcentajes de la siguiente manera Derecho con 25.4 \%, Psicología con 
$25.9 \%$, Comunicación Organizacional con 12.1 $\%$, Negocios y Comercio Internacional con 27.8 $\%$ y Administración con $8.9 \%$; cabe señalar que las carreras de Administración y Comunicación son las carreras que menos alumnos tienen dentro del campus.

Con relación a los semestres, estos corresponden al primer semestre de año en el calendario escolar y se repartió en porcentajes de la siguiente manera: segundo con $31.8 \%$, cuarto con $29.5 \%$, sexto con $21.5 \%$ y octavo con $17.2 \%$. El porcentaje por semestre fue disminuyendo dado que el semestre octavo casi no se encuentra en clases presenciales en el campus; con relación al turno, $51.7 \%$ eran del turno matutino, $45.8 \%$ del vespertino y $2.5 \%$ mencionaron llevar clases en ambos turnos.

Asimismo, la muestra contó con una media de edad de 21.20 años cumplidos y una desviación estándar de 3.55 años, un mínimo de edad 18 años y un máximo de 49 años. Con relación al género, el $66.3 \%$ pertenecían al género femenino y un $33.7 \%$ al género masculino. El $87.6 \%$ presentaron un estado civil de soltero, un $4.5 \%$ eran casados, un $6 \%$ en unión libre, $.4 \%$ separados y un .9\% divorciados. El $55.1 \%$ mencionaron que trabajaban aparte de estudiar; sin embargo, el $60.9 \%$ mencionaron que dependían de sus padres principalmente en el plano económico.

Por último, con relación al rendimiento académico, en su estatus en el kárdex el 84.9 $\%$ mencionaron ser regulares y un $15.1 \%$ en estado irregular. Un 36 \% reportó no haber reprobado nunca alguna materia en lo que va de su trayectoria escolar; se observó que en los rangos de calificaciones de kárdex el $4.1 \%$ se ubica en el de 60 a 70 de calificación, $20.8 \%$ en el de 71 a $80,45.9 \%$ en el de 81 a 90 y $28.9 \%$ en el de 91 a 100.

\section{Instrumentos}

Para detectar problemas de salud mental en la muestra en estudio se utilizó la Escala de Medida de los Síntomas Transversales de Nivel 1 del DSM-5 autoevaluada (DSM) para adulto de la Asociación Psiquiátrica Americana (2014), instrumento validado para este estudio en población sonorense, el cual presentó alta consistencia interna (Alfa de Cronbach = .916). Consta de 37 reactivos en escala Likert y mide 21 indicadores de trastornos mentales; si bien la presencia del indicador no significa sea un diagnóstico de trastorno mental, sí la probabilidad de la presencia del trastorno.

A su vez, para medir la presencia de habilidades de afrontamiento se utilizó el Inventario de Estrategias de Afrontamiento CSI (Tobin et al., 1989) adaptado en población mexicana por Rodríguez et al. (2014). Consta de 41 reactivos en escala Likert, que miden la presencia de 8 habilidades de afrontamiento; para el presente estudio presentó también alta consistencia interna (Alfa de Cronbach $=.895$ ). Por último, el rendimiento académico se midió a través de indicadores como estatus regular e irregular, además del rango de calificaciones presentados en el kárdex de cada estudiante. 


\section{Procedimiento}

El presente proyecto de investigación se realizó con el apoyo directo de recursos PRODEP. Fue evaluado y aprobado por las instancias académicas correspondientes: primeramente, por la Academia de Ciencias Jurídicas y Psicológicas perteneciente al Departamento de Ciencias Sociales del campus; posteriormente, fue revisado por el comité de investigación y ética de la División de Ciencias Administrativas Sociales y Agropecuarias para su posterior aval del Consejo Divisional al que corresponde el comité.

Se reclutó a alumnos de la carrera de Psicología de los semestres cuarto, sexto y octavo para apoyo en la aplicación de los instrumentos de medición; se entrenó al grupo de estudiantes a través de la aplicación del instrumento a ellos mismos, de tal forma que se pudieran resolver las dudas correspondientes. Se consiguieron los permisos correspondientes con los jefes de departamento del campus y la aplicación se realizó en una semana al inicio del semestre para no entorpecer las actividades académicas (ocupando los 5 días tanto en el turno matutino como en el vespertino, el tiempo de aplicación variaba de 45 a 60 minutos dependiendo del estudiante). Se dividió a los estudiantes de Psicología en grupos de 2 personas, las cuales realizaron la aplicación, resolvieron dudas $\mathrm{y}$ revisaron si los instrumentos estuvieron contestados en su totalidad. Posteriormente se estructuró una matriz en SPSS para el vaciado de los datos, que se hizo con el apoyo de los alumnos anteriormente mencionados, para su posterior análisis.

\section{Análisis de datos}

El análisis de datos se realizó a través del paquete estadísticos SPSS en su versión 22. Primeramente, se calculó el nivel de consistencia interna de cada una de las escalas utilizadas, para lo cual se hizo uso del estadístico Alfa de Cronbach, considerando como aceptable que las alfas fueran mayores a 0.7. Posteriormente, se obtuvieron estadísticas univariadas para las variables sociodemográficas: medias y desviaciones estándar para las variables continuas como edad; frecuencias y porcentajes para las variables categóricas como género.

Se utilizó la prueba de normalidad Kolmogorov-Smirnov para comparar la función de distribución acumulada empírica (ECDF) de los datos de la muestra. Los resultados indicaron que el valor $\mathrm{p}(\mathrm{p}>.05)$ fue mayor que el nivel de significancia elegido, por lo cual se aceptó la hipótesis nula y se concluyó que se trata de una distribución normal, ante esto se decidió utilizar estadísticos paramétricos para analizar las diferencias entre grupos (Romero, 2016).

Se promediaron los factores de cada escala utilizada según lo planteado por la base teórica de cada instrumento, utilizando la medida de prevalencia como forma de determinar la presencia del indicador en la población estudiantil. Posteriormente se utilizó la prueba estadística de $\mathrm{T}$ de Student con la corrección estadística de $\mathrm{F}$ de Schneidecor, tomando a consideración una $\mathrm{P}$ 
$<0.05$ para considerar diferencias significativas entre los grupos de rendimiento académico por estatus en el kárdex. Por último, se utilizó el Análisis de Varianza de un Factor (ANOVA) con la prueba Post Hoc HSD de Tukey para realizar comparaciones entre grupo de rangos de calificaciones presentadas en el kárdex.

\section{Resultados}

Las tablas que se presentan a continuación (Tablas 1 a 6) exponen las prevalencias de los indicadores de problemas de salud mental, tanto a nivel campus Nogales, como en cada una de las carreras que se imparten en este. Las tablas se componen de 4 columnas, en las tres primeras se mencionan el grado en que se presenta el indicador: leve, moderado y grave. El grado leve hace referencia de la presencia del problema por periodos espaciados, el grado moderado se refiere a la presencia del problema por periodos más seguidos, aunque aún intermitentes, y el grado grave indica la presencia del problema ya de forma recurrente; además de lo anterior, se presenta una cuarta columna que contiene el total de la prevalencia del indicador en la población estudiantil. Para el presente artículo solo se describirá y mencionará los tres indicadores principales en sus tres grados y el global en la Tabla 1, la cual hace referencia a todo el campus, y solo se hará mención del total en cada una de las carreras. Es importante resaltar que en todas las tablas el grado que presenta mayor porcentaje de prevalencia es el grado leve.
Con relación a la muestra total del campus, en la prevalencia de los indicadores en la población estudiantil (Tabla 1) es el indicador de ira el que presenta mayor prevalencia, lo cual refiere un problema la regulación de emociones en los alumnos del campus; es probable que los alumnos presentan inestabilidad en sus estados de humor, observándose irritables e hipersensibles ante mínimas provocaciones, presentando periodos de explosividad. El segundo indicador más elevado es el de depresión, demostrando la presencia de problemas en el estado de ánimo en 5 de cada 10 alumnos del campus. En tercero está el indicador de síntomas somáticos, lo cual indica que la mitad de los alumnos presenta ansiedad al sentir problemas a nivel fisiológico con probabilidad de que los maximicen.

El panorama se modifica al observar a nivel de grados; en el grado leve, el indicador de ira se presenta con mayor prevalencia, seguido por el indicador de manía, y en tercero por el indicador depresivo, lo cual hace pensar periodos de inestabilidad en los estudiantes, que oscilan entre altas y bajas en su estado de ánimo. En el grado moderado se presenta el indicador de ira con mayor prevalencia, seguido por el indicador de síntomas somáticos, y como tercero el indicador de depresión. Por último, el grado grave o severo se observa que son los indicadores de problemas de sueño los de mayor prevalencia, seguidos por el indicador de ira, y en tercero el indicador de problemas de memoria. 
Con relación a los comportamientos suicidas, son los comportamientos parasuicidas como las autolesiones las que se presentan con mayor prevalencia. Se presentaron en todas las carreras, en promedio, en 2 de cada
10 estudiantes, a excepción de la carrera de Negocios donde ocurrió en 1 de cada 10, presentando mayor prevalencia en la carrera de Psicología.

\section{Tabla 1}

Prevalencia en el campus de indicadores de problemas de salud mental

\begin{tabular}{|c|c|c|c|c|}
\hline Indicador & Leve & Moderado & Grave & Tota \\
\hline Depresión & 27.9 & 16.7 & 6.3 & 50.9 \\
\hline Ira & 28.5 & 21.9 & 11.8 & 62.2 \\
\hline Manía & 28.1 & 13.4 & 3.6 & 45.1 \\
\hline Ansiedad & 20.9 & 11.5 & 2.9 & 35.3 \\
\hline Síntomas somáticos & 24.2 & 17.3 & 8 & 49 \\
\hline Psicosis & 5.3 & 2.4 & 1.3 & 9 \\
\hline Problemas de sueño & 18.8 & 15.4 & 13.3 & 47.5 \\
\hline Problemas de memoria & 15.8 & 13.8 & 9.2 & 38.8 \\
\hline Pensamientos obsesivos & 17.7 & 6.7 & 4 & 28.4 \\
\hline Disociación & 17.3 & 9.4 & 8.3 & 35 \\
\hline Disfunción de la personalidad & 19.8 & 11.8 & 5.8 & 37.4 \\
\hline Consumo de sustancias & 8.6 & 2.8 & 1.5 & 12.9 \\
\hline Maltrato doméstico & 3.8 & 2.2 & 1 & 7 \\
\hline Hiperactividad e impulsividad & 10.4 & 4.2 & 2.6 & 17.2 \\
\hline Fobia social & 16.3 & 9.2 & 4.9 & 23.1 \\
\hline Problemas alimentarios & 13.9 & 6.8 & 1.9 & 22.6 \\
\hline Ideación suicida & 5.9 & 0 & 0 & 5.9 \\
\hline Intento suicida & .6 & 0 & .1 & .7 \\
\hline Autolesiones & 4.2 & 6.3 & 6.1 & 16.6 \\
\hline
\end{tabular}

Nota: los valores están expresados en porcentajes (\%) 
En la Tabla 2, se muestra las prevalencias presentadas por los estudiantes de la carrera de Psicología; en el total de prevalencia de indicadores presentado por la carrera es el indicador de ira el de mayor prevalencia, seguido por el de síntomas somáticos y en tercer lugar los problemas de sueño. Esto indica que los alumnos de psicología pudieran presentar inestabilidad en sus emociones, somatizaciones físicas como forma de descargar el estrés y dificultades para conciliar una adecuada calidad de sueño al dormir.

\section{Tabla 2}

Prevalencia en la carrera de Psicología de indicadores de problemas de salud mental

\begin{tabular}{|c|c|c|c|c|}
\hline Indicador & Leve & Moderado & Grave & Total \\
\hline Depresión & 24.7 & 20.2 & 4.5 & 49.4 \\
\hline Ira & 30.3 & 20.8 & 11.2 & 62.3 \\
\hline Manía & 24.7 & 15.7 & 3.4 & 43.8 \\
\hline Ansiedad & 23.1 & 14 & 1.7 & 38.8 \\
\hline Síntomas somáticos & 21.3 & 23.6 & 6.7 & 51.6 \\
\hline Psicosis & 5.6 & 2.7 & 1.1 & 9.4 \\
\hline Problemas de sueño & 19.1 & 16.3 & 15.2 & 50.6 \\
\hline Problemas de memoria & 12.9 & 16.3 & 10.7 & 39.9 \\
\hline Pensamientos obsesivos & 16.8 & 9.5 & 2.8 & 29.1 \\
\hline Disociación & 18 & 9 & 7.9 & 34.9 \\
\hline Disfunción de la personalidad & 19.8 & 11.8 & 5.8 & 37.4 \\
\hline Consumo de sustancias & 8.3 & 2.7 & .6 & 11.6 \\
\hline Maltrato doméstico & 3.3 & 2.7 & 1.1 & 7.1 \\
\hline Hiperactividad e impulsividad & 10.1 & 5 & 2.2 & 17.3 \\
\hline Fobia social & 24 & 8.4 & 7.3 & 39.7 \\
\hline Problemas alimentarios & 11.2 & 8.4 & 2.2 & 21.8 \\
\hline Ideación suicida & 5 & 0 & 0 & 5 \\
\hline Intento suicida & 1.1 & 0 & 0 & 1.1 \\
\hline Autolesiones & 7.3 & 9 & 7.3 & 23.6 \\
\hline
\end{tabular}

Nota: los valores están expresados en porcentajes (\%) 
En la Tabla 3 se presentan los resultados de la carrera de Derecho. En el total de indicadores la prevalencia más alta fue del indicador de ira, seguido por el indicador de depresión y en tercero el indicador de manía, por lo que se probabiliza que los estudiantes presenten inestabilidad en sus emociones, sobre todo al momento de regular su estado de ánimo, que es probable oscile en periodos de disforia a euforia.

\section{Tabla 3}

Prevalencia en la carrera de Derecho de indicadores de problemas de salud mental

\begin{tabular}{|c|c|c|c|c|}
\hline Indicador & Leve & Moderado & Grave & Total \\
\hline Depresión & 28 & 11.4 & 8.6 & 48 \\
\hline Ira & 26.9 & 21.1 & 13.7 & 61.7 \\
\hline Manía & 31.8 & 9.1 & 5.7 & 46.6 \\
\hline Ansiedad & 21.1 & 9.6 & 4 & 34.7 \\
\hline Síntomas somáticos & 22.2 & 13.2 & 10.9 & 46.3 \\
\hline Psicosis & 4 & 2.7 & 2.3 & 9 \\
\hline Problemas de sueño & 19.4 & 12.6 & 12.6 & 44.6 \\
\hline Problemas de memoria & 18.9 & 10.3 & 6.9 & 36.1 \\
\hline Pensamientos obsesivos & 20 & 4.6 & 2.9 & 27.5 \\
\hline Disociación & 16 & 7.4 & 9.7 & 33.1 \\
\hline Disfunción de la personalidad & 13.2 & 13.2 & 5.1 & 31.5 \\
\hline Consumo de sustancias & 10.3 & 2.2 & 1.7 & 14.2 \\
\hline Maltrato doméstico & 5.1 & 2.7 & 1.7 & 9.5 \\
\hline Hiperactividad e impulsividad & 9.8 & 2.8 & 3.4 & 16 \\
\hline Fobia social & 13.2 & 10.9 & 2.9 & 27 \\
\hline Problemas alimentarios & 16.6 & 5.1 & 2.3 & 24 \\
\hline Ideación suicida & 6.9 & 0 & 0 & 6.9 \\
\hline Intento suicida & 0 & 0 & 0 & 0 \\
\hline Autolesiones & 4 & 6.3 & 5.1 & 15.4 \\
\hline
\end{tabular}

Nota: los valores están expresados en porcentajes (\%) 
Con relación a la Tabla 4, donde se muestran los indicadores presentados por la carrera de Comunicación Organizacional, en la totalidad de prevalencias de los indicadores se observa que es el indicador de ira el que presenta mayor porcentaje. Describe que 7 de cada 10 estudiantes de esta carrera presentan inestabilidad emocional, seguido por el indicador de depresión, se señala que 6.6 estudiantes de cada 10 en esta carrera presentan algún tipo de problema depresivo, $\mathrm{y}$ por tercero el indicador de síntomas somáticos con 6 de cada 10 estudiantes, lo cual hace referencia a problemas en la regulación de sus emociones y problemas en la descarga adecuada de la tensión y estrés.

\section{Tabla 4}

Prevalencia en la carrera de Comunicación Organizacional de indicadores de problemas de salud mental

\begin{tabular}{lcccc}
\hline Indicador & Leve & Moderado & Grave & Total \\
\hline Depresión & 36.2 & 24.1 & 6 & 66.3 \\
Ira & 37.3 & 22.9 & 12 & 72.2 \\
Manía & 26.5 & 15.6 & 3.6 & 45.7 \\
Ansiedad & 18 & 19.2 & 3.6 & 40.8 \\
Síntomas somáticos & 26.5 & 21.7 & 10.8 & 59 \\
Psicosis & 7.2 & 4.8 & 1.2 & 13.2 \\
Problemas de sueño & 12 & 19.3 & 13.2 & 44.5 \\
Problemas de memoria & 12 & 13.3 & 18.1 & 43.4 \\
Pensamientos obsesivos & 15.6 & 8.4 & 4.8 & 28.8 \\
Disociación & 18.1 & 10.8 & 4.8 & 23.7 \\
Disfunción de la personalidad & 25.3 & 12 & 10.8 & 48.1 \\
Consumo de sustancias & 15.6 & 4.8 & 2.4 & 22.8 \\
Maltrato doméstico & 2.4 & 6 & 1.2 & 9.6 \\
Hiperactividad e impulsividad & 14.4 & 4.8 & 4.8 & 24 \\
Fobia social & 16.8 & 9.6 & 7.2 & 33.6 \\
Problemas alimentarios & 16.8 & 10.8 & 2.4 & 30 \\
Ideación suicida & 3.6 & 0 & 0 & 3.6 \\
Intento suicida & 1.2 & 0 & 0 & 1.2 \\
Autolesiones & 4.8 & 6 & 7.2 & 18 \\
\hline
\end{tabular}

Nota: los valores están expresados en porcentajes (\%) 
La Tabla 5 muestra los indicadores presentados por la carrera de Administración, en el total de prevalencia el primero es el indicador de ira, seguido por el indicador de problemas de sueño y en tercero el indicador de depresión, además de síntomas somáticos. Lo cual indica también inestabilidad en las emociones en los estudiantes de esta carrera, que al igual que en Comunicación 7 de cada 10 estudiantes presentará estas características. Es importante resaltar que las carreras de Comunicación Organizacional y Administración sobrepasaron en sus prevalencias totales la prevalencia general del campus en los indicadores de problemas de salud mental, en comparación con las otras tres carreras.

\section{Tabla 5}

Prevalencia en la carrera de Administración de indicadores de problemas de salud mental

\begin{tabular}{lcccc}
\hline Indicador & Leve & Moderado & Grave & Total \\
\hline Depresión & 21.3 & 18.1 & 16.4 & 55.8 \\
Ira & 26.2 & 29.5 & 16.4 & 72.1 \\
Manía & 26.1 & 8.2 & 4.9 & 39.2 \\
Ansiedad & 18 & 8.2 & 9.8 & 36 \\
Síntomas somáticos & 32.8 & 14.7 & 8.2 & 55.7 \\
Psicosis & 3.3 & 1.6 & 3.3 & 8.2 \\
Problemas de sueño & 21.3 & 19.7 & 16.4 & 57.4 \\
Problemas de memoria & 13.1 & 19.7 & 8.2 & 41 \\
Pensamientos obsesivos & 18 & 13.1 & 6.6 & 37.7 \\
Disociación & 23 & 11.5 & 14.8 & 49.3 \\
Disfunción de la personalidad & 18.1 & 16.4 & 8.2 & 42.7 \\
Consumo de sustancias & 8.2 & 4.8 & 3.3 & 16.3 \\
Maltrato doméstico & 1.6 & 0 & 1.6 & 3.2 \\
Hiperactividad e impulsividad & 6.6 & 3.2 & 6.6 & 16.4 \\
Fobia social & 14.7 & 8.2 & 8.2 & 31.1 \\
Problemas alimentarios & 13.1 & 8.1 & 0 & 21.2 \\
Ideación suicida & 11.5 & 0 & 0 & 11.5 \\
Intento suicida & 0 & 0 & 0 & 0 \\
Autolesiones & 3.3 & 8.2 & 6.6 & 18.1 \\
\hline
\end{tabular}

Nota: los valores están expresados en porcentajes (\%) 
La Tabla 6 expone las prevalencias de la carrera de Negocios y Comercio Internacional, es esta carrera la que presentó prevalencias menores en comparación de las otras; no obstante, sigue presentando porcentajes significativos. Con relación a las prevalencias generales en porcentaje de presencia, se observa que, en el total prevalente, los indicadores de ira, problemas de sueño y manía ocupaban en ese orden los mayores porcentajes.

\section{Tabla 6}

Prevalencia en la carrera de Negocios de indicadores de problemas de salud mental

\begin{tabular}{lcccc}
\hline Indicador & Leve & Moderado & Grave & Total \\
\hline Depresión & 19.3 & 14.7 & 3.1 & 37.1 \\
Ira & 25.1 & 20.9 & 8.9 & 54.9 \\
Manía & 26.7 & 15.7 & 1.6 & 44 \\
Ansiedad & 20.4 & 8.3 & 5 & 29.2 \\
Síntomas somáticos & 24.6 & 14.1 & 5.2 & 33.4 \\
Psicosis & 6.3 & 2.6 & 0 & 8.9 \\
Problemas de sueño & 19.9 & 14.1 & 11.5 & 45.5 \\
Problemas de memoria & 18.3 & 13.1 & 6.3 & 37.7 \\
Pensamientos obsesivos & 17.3 & 3.1 & 2.6 & 23 \\
Disociación & 15.7 & 10.5 & 6.8 & 33 \\
Disfunción de la personalidad & 21.5 & 10 & 3.7 & 35.2 \\
Consumo de sustancias & 5.2 & 2.6 & 1 & 8.8 \\
Maltrato doméstico & 4.1 & 2 & 0 & 6.2 \\
Hiperactividad e impulsividad & 11 & 4.7 & 6.6 & 16.4 \\
Fobia social & 13 & 7.8 & 2.6 & 23.4 \\
Problemas alimentarios & 13.1 & 4.2 & 1.6 & 18.9 \\
Ideación suicida & 4.7 & 0 & 0.9 & 4.7 \\
Intento suicida & .5 & 0.1 & .5 & 1 \\
Autolesiones & 1.6 & 5.2 & 0.9 \\
\hline
\end{tabular}

Nota: los valores están expresados en porcentajes (\%) 
La Tabla 7 expone los resultados de los indicadores de la presencia de habilidades de afrontamiento en los estudiantes, tanto a nivel global como por carrera. A título informativo, las ocho estrategias de afrontamiento presentadas diferencian un estilo de afrontamiento activo o dirigidas a la acción, tales como resolución de problemas, reestructuración cognitiva, expresión emocional y apoyo social, en contraste con las estrategias de afrontamiento pasivo, no dirigidas a la acción, tales como evitación de problemas, pensamiento desiderativo, autocrítica, y retirada social. Se observa en los resultados que las mayores prevalencias se encuentran en aquellas habilidades de afrontamiento pasivo, en todas las carreras, siendo la habilidad de resolución de problemas (habilidad de afrontamiento orientada a la acción) la que presenta menor prevalencia tanto a nivel global como por carrera.

\section{Tabla 7}

Prevalencia de indicadores de habilidades de afrontamiento por carrera y campus

\begin{tabular}{lcccccc}
\hline Habilidad & Nog & LPS & LP & LCO & LA & LNCl \\
\hline Resolución de problemas & 58.8 & 56.3 & 60.8 & 67.1 & 54.2 & 57.1 \\
Autocrítica & 93.6 & 93.2 & 90.6 & 92.6 & 95 & 96.8 \\
Expresión emocional & 81.4 & 83 & 79.2 & 92.4 & 70.7 & 80.3 \\
Pensamiento desiderativo & 67.2 & 70.2 & 60.5 & 75.6 & 68.4 & 65.1 \\
Apoyo social & 65.5 & 64.4 & 63.5 & 72.5 & 62.3 & 66.5 \\
Restructuración cognitiva & 71.9 & 73.3 & 73.1 & 70.7 & 67.8 & 71.5 \\
Evitación de problemas & 94.6 & 92.5 & 95.7 & 95.1 & 96.7 & 94.6 \\
Retirada social & 100 & 100 & 100 & 100 & 100 & 100 \\
\hline
\end{tabular}

Nog: Nogales; LPS= Psicología; LD= Derecho; LCO= Comunicación Organizacional; LA= Administración; LNCI= Negocios y Comercio Internacional.

Nota: los valores están expresados en porcentajes (\%)

Las Tablas 8 y 9 presentan los resultados del estadístico $\mathrm{T}$ de Student, con relación a la diferencia de medias, para determinar si existen o no diferencias entre los indicadores de salud mental y habilidades de afrontamiento con el rendimiento académico. Se observa en la Tabla 8, con relación a los indicadores de salud mental, que solo los indicadores de depresión 
y problemas de sueño presentan diferencias significativas entre el grupo de alumnos regulares e irregulares, siendo el grupo de alumnos irregulares el de mayor problemática en los indicadores antes mencionados.

\section{Tabla 8}

Diferencia de medias entre alumnos con estatus regular e irregular en indicadores de salud mental

\begin{tabular}{lcccccc}
\hline \multicolumn{1}{c}{ Indicador } & Regular & Irregular & $\mathrm{F}$ & $\mathrm{Sig}$ & $\mathrm{t}$ & $\mathrm{gl}$ \\
\hline Depresión & 2.72 & 2.94 & .456 & .032 & -2.19 & 75.68 \\
Ira & 2.69 & 2.88 & .735 & .077 & -.1 .78 & 94.39 \\
Manía & 2.61 & 2.64 & .542 & .766 & -.299 & 68.12 \\
Ansiedad & 2.71 & 2.81 & .023 & .317 & -1.00 & 59.48 \\
Síntomas somáticos & 2.82 & 2.94 & .193 & .660 & -1.17 & 83.25 \\
Psicosis & 2.67 & 2.85 & .594 & .474 & -.735 & 13.79 \\
Problemas de sueño & 2.85 & 3.78 & 8.63 & .015 & -2.44 & 324 \\
Problemas de memoria & 2.79 & 2.97 & .436 & .185 & -1.34 & 58.48 \\
Pensamientos obsesivos & 2.58 & 2.76 & .893 & .190 & -1.33 & 45.84 \\
Disociación & 2.71 & 2.84 & .404 & .336 & -.912 & 53.90 \\
Disfunción de la personalidad & 2.80 & 2.80 & .075 & .985 & .018 & 62.33 \\
Consumo de sustancias & 2.60 & 2.75 & 4.71 & .341 & -.958 & 86 \\
Maltrato doméstico & 2.74 & 3.09 & 1.82 & .190 & -1.34 & 24.61 \\
Hiperactividad e impulsividad & 2.67 & 2.69 & .705 & .902 & -.124 & 41.75 \\
Fobia social & 2.82 & 2.94 & 1.78 & .422 & -.810 & 44.25 \\
Problemas alimentarios & 2.72 & 2.88 & .477 & .297 & -1.06 & 30.61 \\
\hline
\end{tabular}

Nota: Sig. $P<.05$

La Tabla 9 presenta la diferencia de medias de los indicadores de afrontamiento, solo la habilidad de restructuración cognitiva presentó una diferencia significativa, siendo el grupo de alumnos regulares el que mayor presencia tiene de la habilidad de desarrollo de esta estrategia cognitiva para modificar el significado de la situación estresante. 


\section{Tabla 9}

Diferencia de medias en alumnos con estatus regular e irregular en indicadores de afrontamiento

\begin{tabular}{lcccccc}
\hline Habilidad & Regular & Irregular & $\mathrm{F}$ & $\mathrm{Sig}$ & $\mathrm{t}$ & $\mathrm{gl}$ \\
\hline Resolución de problemas & 17.89 & 17.14 & .861 & .167 & 1.39 & 136.33 \\
Autocrítica & 13.25 & 13.73 & .111 & .428 & -.795 & 137.77 \\
Expresión emocional & 15.64 & 15.18 & .857 & .431 & -.790 & 133.51 \\
Pensamiento desiderativo & 17.20 & 17.73 & 1.94 & .501 & -.676 & 117.45 \\
Apoyo social & 16.26 & 15.27 & .034 & .129 & 1.52 & 140.36 \\
Restructuración cognitiva & 16.18 & 14.81 & .002 & .012 & 2.54 & 135.64 \\
Evitación de problemas & 13.68 & 13.26 & .153 & .373 & .894 & 131.41 \\
Retirada social & 13.18 & 13.73 & .241 & .333 & -.973 & 130.18 \\
\hline
\end{tabular}

Nota: Sig. $p<.05$

Por último, aunque no se presenta en forma de tabla, se hace mención de los resultados presentados por el análisis de varianza (ANOVA de un solo factor), en el cual se hicieron comparaciones de rangos de calificaciones de los estudiantes a nivel de todo el campus.

Se observaron diferencias significativas $(\mathrm{p}<.05)$ entre el rango 71 a 80 y el rango 91 a 100 en los indicadores de problemas de salud mental de pensamientos obsesivos $(\mathrm{p}=.032)$, consumo de sustancias $(\mathrm{p}=.000)$, ansiedad $(\mathrm{p}$ $=.049)$, psicosis $(\mathrm{p}=.034)$, problemas de sueño $(p=.017)$, pensamientos obsesivos $(p=.032)$, consumo de sustancias $(\mathrm{p}=.000)$, maltrato $(\mathrm{p}=$ $.038)$ e hiperactividad $(p=.025)$. Se encontraron diferencias entre el rango 71 a 80 y el rango 81 a 90 en el indicador manía $(\mathrm{p}=.011)$; a su vez los estudiantes con rango de 61 a 70 presentaron mayor promedio en comparación con los estudiantes de rango 81 a 90 con el indicador de maltrato $(\mathrm{p}=.004)$ y con el rango 91 a 100 en el indicador de hiperactividad $(\mathrm{p}=.038)$; por último, el rango de 81 a 90 presentó diferencia en el indicador hiperactividad en comparación con los estudiantes de rango 91 a $100(\mathrm{p}=.025)$.

De igual forma se observaron diferencias $(\mathrm{p}<.05)$ en los indicadores de autocrítica $(\mathrm{p}=$ $.030)$, apoyo social $(\mathrm{p}=.019)$ y restructuración cognitiva $(p=.029)$, en los estudiantes con rango de calificaciones de 71 a 80 en comparación con los estudiantes de rango de 91 a 100. Los estudiantes de rango 61 a 70 presentaron menor orientación de búsqueda de apoyo emocional apoyo social $(\mathrm{p}=.040)$ en comparación con aquellos que estaban en el rango 91 a 100. 


\section{Discusión}

El conocer los estados de salud mental en poblaciones específicas es importante, dado que es una base para prevenir problemas de esta índole y generar estrategias de intervención con tiempo, de tal forma que se mejore el bienestar no solo del individuo, sino a nivel familiar y comunitario(Posada, 2013). En las universidades no debe pasar desapercibido, ya que es necesario tener actualizada la información del estado de salud mental de sus estudiantes, fortaleciendo el área y mejorando sus habilidades (Mitchel et al., 2016), de tal modo que impacte de forma positiva en el aprendizaje (Sánchez et al., 2015).

La presente investigación presentó evidencia tanto de una alta prevalencia de indicadores que refieren la posibilidad de la presencia de desórdenes o trastornos mentales, como una alta prevalencia de indicadores de habilidades de afrontamiento inadecuadas $o$ inefectivas en los estudiantes de UNISON campus Nogales. Por ello, puede afirmarse que se presentan problemas de salud mental en la población en estudio, respondiendo así la primera pregunta de investigación y cumpliendo el primer objetivo general.

Es importante aclarar que los resultados obtenidos no son sinónimo de diagnóstico clínico, sin embargo, las puntuaciones altas indican que existen síntomas problemáticos y significativos, y que es necesario realizar una evaluación más profunda y exhaustiva, así como un posterior tratamiento y seguimiento; lo anterior explica por qué en los estudios revisados los porcentajes fueron menores (Gallagher, 2014; Micin y Bagladi, 2011;). No obstante, los resultados presentes pueden guiar la toma de decisiones, dado que son una referencia directa de cómo el estudiante se percibe a sí mismo y de que el problema está presente en la población estudiada.

Los resultados indican la presencia de malestar psicológico a nivel cognitivo, emocional y comportamental en los estudiantes, y esto se presenta en todas las carreras y a nivel campus, siendo las carreras de Comunicación Organizacional y Administración las que presentan mayor prevalencia del problema en gran parte de los indicadores evaluados. Esta situación abre una nueva interrogante: ¿por qué esas carreras presentan mayor problemática?, lo cual representa un área de oportunidad para futuros trabajos.

Los resultados concuerdan con lo encontrado en investigaciones anteriores en ciertos indicadores como lo son los problemas de regulación e inestabilidad emocional (Pozos et al., 2014), problemas del estado de ánimo como depresión(Gallagher, 2014; Lester, 2014; Lipson et al. 2015), problemas de sueño (Valerio et al., 2016), así como de conductas autodestructivas como lo son los comportamientos suicidas (Lester, 2014; Lipson et al., 2015). En otros indicadores se encontró una baja en prevalencia, como lo es el indicador de ansiedad, referido en algunas investigaciones (Micin y Bagladi, 2011), lo cual resultó no ser tan significativo en comparación con otros. Indicadores como 
lo son la somatización, manía, disociación y la disfunción de la personalidad, y problemas de memoria presentan poca evidencia empírica, por lo que constituye un área de oportunidad para seguir investigando.

En relación con los indicadores de afrontamiento, diversos estudios han explorado el rol del afrontamiento como método para resolver estados de tensión emocional no solo a nivel universitario (Díaz, 2010). La presente investigación encontró que los estudiantes del campus se orientan a un predominio en el uso del estilo de afrontamiento pasivo (Sepúlveda et al., 2012). Este se asocia con menores niveles de bienestar (Byrd y McKinney, 2012; Chao, 2011) y probabiliza que los problemas de salud mental no solo se presenten, sino que se mantengan con el tiempo (Mahmoud et al., 2012), lo cual pudiera afectar la capacidad de adaptación en el estudiante (Pritchard et al., 2007; Restrepo y Jaramillo, 2012). Es importante entender por qué se presenta de esta manera y las implicaciones teóricas que pudiera tener el poder encontrar aquellas variables asociadas al déficit en habilidades, campo abierto para futuros trabajos de investigación.

Los estudiantes presentaron alta prevalencia en estrategias basadas en la autoinculpación y la autocrítica; la negación y evitación de pensamientos o actos relacionados con el acontecimiento estresante y principalmente el aislamiento, estas dos últimas como habilidades o estrategias dirigidas a controlar las emociones y que probabilizan que en situaciones de alta ten- sión presenten problemas de somatización como forma de liberar la emoción (Gustems y Calderón, 2013). A su vez, la presencia de una baja en aquellas habilidades de apoyo social y un alta en conductas de aislamiento, convergen como menciona el Modelo General de Salud Mental (Barrón y Sánchez, 2001) y explica por qué se presenta un alta en los indicadores de problemas de salud mental o deterioro psicológico.

La etapa universitaria está caracterizada por presentar situaciones de alta tensión $\mathrm{y}$ demanda al estudiante en diversos niveles (Antuñez y Vinet, 2013; Badder et al., 2016; García et al., 2012; Pozos et al., 2015;), eventos que probabilizan la presencia de problemas de salud mental tanto a nivel psicológico como comportamentales (Pozos et al., 2014). Sin embargo, no se puede establecer que la vida universitaria sea el único factor que probabilice la presencia de esta clase de problemas, ya que no necesariamente el estudiante interactúa solo en el mundo universitario, sino que se desenvuelve en otros contextos como lo son el entorno familiar y el entorno laboral, los cuales también generan al individuo diversas demandas que deben ser resueltas. Estas, al conjuntarse con el estrés académico, aumentan la probabilidad de presencia de esta clase de problemas, por lo que es importante continuar con el estudio de esas variables para profundizar no solo en factores de riesgo, sino también en factores protectores.

Con relación a la segunda pregunta de investigación y segundo objetivo, en el análisis de medias se observó diferencias solo 
en dos indicadores de salud mental, que fueron depresión y problemas de sueño, y que estos se presentan con una media mayor en los alumnos con estatus de irregulares; además, la habilidad de reestructuración cognitiva se presenta mayormente en los alumnos regulares. A su vez, se observó que los alumnos de bajos promedios (rangos de 61 a 70 y 71 a 80) presentan mayores problemas en algunos indicadores de salud mental como lo son los pensamientos obsesivos, psicosis, ansiedad y problemas de sueño, y menor uso de estrategias como lo son apoyo social o reestructuración cognitiva, y la tendencia a la autocrítica.

Por lo anterior, se puede determinar que los problemas de salud mental pudieran estar asociados a una baja en el rendimiento académico en el estudiante (Barraza, 2006). Sin embargo, aunque los resultados indiquen diferencias, no son concluyentes, dado que se necesita otro tipo de estrategias de análisis para medir la asociación, el efecto e impacto de estas variables sobre el rendimiento, y un mayor control de variables extrañas para evitar sesgos al momento de interpretar los resultados, lo cual presenta un área de trabajo que aún está abierta.

Dentro de las limitaciones del presente estudio, se encuentra no haber abarcado el $100 \%$ del alumnado. Esta situación pudiera solventarse en futuras aplicaciones ampliando el tiempo del proceso de aplicación y aplicando otro tipo de estrategias de control de valores perdidos (Montenegro et al., 2015).
Con los resultados obtenidos, se contribuye a un mayor conocimiento sobre problemáticas de salud mental en el campus. A su vez, se sienta una base teórica para el diseño de estrategias preventivas a través de dos líneas de acción que deben ser abordadas de forma inmediata: 1) la disminución de los indicadores que probabilizan la presencia de trastornos mentales y 2) el aumento de estrategias o habilidades de afrontamiento del estudiante a los problemas de la vida. Además, esta base teórica también sirve como fundamento para un posterior estudio, de tal forma que permita estudiar la evolución de los problemas e ir ajustando las estrategias con relación a lo que se fuere presentando, tanto a nivel preventivo como de rehabilitación.

\section{Referencias}

Antunez, Z. y Vinet, E. (2013). Problemas de salud mental en estudiantes de universidad regional chilena. Revista Médica de Chile, 141, 209-216. http://doi.org/10.4067/ S0034-98872013000200010

Asociación Psiquiátrica Americana. (2014). Manual de diagnóstico y estadístico de los trastornos mentales DSM- $V$. Editorial Médica Panamericana.

Baader, T., Rojas, C., Molina, J., Gotelli, M., Álamo, C., Fierro, C. Venezian, S. y Dittus, P. (2014) Diagnostico de la prevalencia de trastornos de la salud mental en estudiantes universitarios $\mathrm{y}$ factores de riesgo emocionales asociados. Revista Chilena de Neuro-psiquiatría, 52 
(523), 167-176. http://doi.org/10.4067/ S0717-92272014000300004

Barraza, A. (2006). Un modelo conceptual para el estudio del estrés académico. Revista PsicologiaCientifica.com, 8(17). http:// www.psicologiacientifica.com/estresacademico-modelo-conceptual

Barrón, A. y Sánchez, E. (2001). Estructura social, apoyo social y salud mental. Psicothema, 13(1), 17-23. http://www. psicothema.com/pdf/408.pdf

Berenzon, S., Saavedra, N., Medina, M., Aparicio, V. y Galván, J. (2013) Evaluación del sistema de salud mental en México: ¿hacia dónde encaminar la atención? Revista Panamericana de Salud Pública, 33(4), 252-8.

Byrd, D. y McKinney, K. (2012). Individual, interpersonal, and institutional level factors associated with mental health of college students. Journal of American College Halth, 60(3), 185-193. https://doi. org/10.1080/07448481.2011.584334

Caso, J. y Hernández, L. (2010). Modelo explicativo del bajo rendimiento escolar: un estudio con adolescentes mexicanos. Revista Iberoamericana de Evaluación Educativa, 3(2), 145-159.

Cassaretto, M. (2010). Relaciones entre la personalidad $y$ el afrontamiento en estudiantes preuniversitarios. Revista Vanguardia Psicológica, 1(1); 202225. https://dialnet.unirioja.es/descarga/ articulo/4815128.pdf
Chao, R. (2011). Managing stress and maintaining well-being: social support, problem-focused coping, and avoidant coping. Journal of Counseling \& Development, 89, 338-348. https://doi. org/10.1002/j.1556-6678.2011.tb00098.x

Chau, C. y Vilela, P. (2017). Variables asociadas a la salud física y mental percibida por estudiantes universitarios de Lima. Liberabit, 23(1), 89-102. http://doi. org/10.24265/liberabit.2017.v23n1.06

Díaz,Y.(2010) Estrés académicoy afrontamiento en estudiantes de Medicina. Revista Humanidades Medicas, 10(1). http:// scielo.sld.cu/pdf/hmc/v10n1/hmc070110. pdf

El Ansari, W., Oskrochi, R. y Haghgoo, G. (2014). Are student's symptoms and health complaints associated with perceived stress at university? Perspectives from the United Kingdom and Egypt. International Journal of Environmental Research and Public Health, 11(10), 9981-10002. https://doi.org/10.3390/ijerph111009981

Ferrel, F., Vélez, J. y Ferrel L. (2014) Factores psicológicos en adolescentes escolarizados con bajo rendimiento académico: depresión y autoestima. Revista Encuentros 12(2), 35-47. http:// doi.org/ 0.15665/re.v12i2.268

Gallagher, R. (2014). National survey of counseling center directors. The International Association of Counseling Services, Inc., Monograph Series Number 9V. 
http://d-scholarship.pitt.edu/28178/1/survey_2014.pdf

García, R., Pérez, F., Pérez, J. y Natividad, L.(2012). Evaluación del estrés en estudiantes de nueva incorporación a la universidad. Revista Latinoamericana de Psicología, 44(2), 143-154.

Gustems, J. y Calderón, C. (2013). Empathy and coping strategies as predictors of wellbeing in Spanish University Students. Electronic Journal of Research in Educational Psychology, 12(1), 129-146. https://doi.org/10.14204/ejrep.32.13117

Jurado, M. (2016). La situación, evolución y composición de las familias vulnerables y su socialización primaria en Matamoros Tamaulipas. Región y Sociedad 28(65), 81-108. http://www.scielo.org. $\mathrm{mx} / \mathrm{pdf} / \mathrm{regsoc} / \mathrm{v} 28 \mathrm{n} 65 / 1870$-3925regsoc-28-65-00081.pdf

Lazarus, R. y Folkman, S. (1986). Estrés y procesos cognitivos. Martínez Roca.

Lester, D. (2014). College student stressors, depression, and suicidal ideation. Psychological Reports, 114(1), 293-296. http:// doi.org/10.2466/12.02.PR0.114k10w7

Lipson, S., Gaddis, S., Heinze, J., Beck, K. y Eisenber, D. (2015). Variations in student mental health and treatment utilization across US colleges and universities. Journal of American College Health, 63(6), 388-396. http://doi.org/10.1080/07 44848.2015.1040411
Mahmoud, J., State, R., Hall, L. y Lennie, T. (2012). The relationship among young adult college students' depression, anxiety, stress, demographics, life satisfaction, and coping styles. Issues in Mental Health in Nursing, 33, 149-156. https://doi.org/10.3 109/01612840.2011.632708

Marenco, A., Suárez, Y. y Palacio, J. (2017). Burnout académico y síntomas relacionados con problemas de salud mental en universitarios colombianos. Psychologia, 11(2), 45-55. http://doi. org/10.21500/19002386.2926

Micin, S. y Bagladi, V. (2011). Salud mental en estudiantes universitarios: incidencia de psicopatología y antecedentes de conducta suicida en población que acude a un servicio de salud estudiantil. Terapia Psicológica 29(1), 53-64. http://doi. org/10.4067/S0718-48082011000100006

Mitchel, J., Reason, R., Hemer, K. y Finley, A. (2016). Perceptions of Campus Climates for Civic Learning as Predictors of College Students. Mental Health. Journal of College and Character, 17(1), 40-52. http://doi.org/10.1080/2194587X.2015. 1125367

Montenegro, E., Oh, Y. y Chesnut, S. (2015). No le tema a los datos perdidos: enfoques modernos para el manejo de datos perdidos. Actualidades en Psicología, 29(119), 2015, 29-42. http://dx.doi. org/10.15517/ap.v29i119.18812 
Organización Mundial de la Salud. (2018).

Salud mental: fortalecer nuestra respuesta. https://www.who.int/es/news$\mathrm{room} /$ fact-sheets/detail/mental-healthstrengthening-our-response

Organización Mundial de la Salud. (2019). Trastornos mentales. https://www.who. int/es/news-room/fact-sheets/detail/ mental-disorders

Pérez, C., Bonnefoy, C. y Cabrera, A. (2011). Análisis, desde la psicología positiva, de la salud mental en alumnos universitarios de primer año de Concepción (Chile). Avances en Psicología Latinoamericana, 29(1), 148-160.

Prado, C. Salazar, Z. Félix, J. (2015) La inteligencia emocional y el rendimiento académico en estudiantes de la escuela profesional de contabilidad. UCV- Hacer Revista de Investigación y Cultura, 4(2), 30-36.

Pritchard, M., Wilson G. y Yamnitz, B. (2007). What predicts adjustment among college students? A longitudinal panel study. Journal of American College Health, 56(1), 15-21. https://doi.org/10.3200/ JACH.56.1.15-22

Posada, J. (2013) La salud mental en Colombia. Biomédica, Instituto Nacional de Salud, 33(4).

Pozos, B., Preciado, M., Plascencia, A., Acosta, M. y Aguilera, M. (2015). Estrés académico y síntomas físicos, psicológicos y comportamentales en estudiantes mexicano de una universidad pública. Ansiedad y estrés, 21(1), 35-42.

Pozos, B., Preciado, M., Acosta, M., Aguilera, M. y Delgado, D. (2014). Academic stress as a predictor of chronic stress in university students. Psicología Educativa, 20(1), 4752. http://doi/10.1016/j.pse.2014.05.006

Restrepo, D. y Jaramillo, J. (2012) Concepciones de la salud mental en el campo de la salud pública. Revista Facultad Nacional de Salud Pública, 30(2), 202-211.

Rodríguez, F., Estrada, C., Rodríguez, F. y Bringas, C. (2014). Adaptación del inventario de estrategias de afrontamiento (CSI) a la población penitenciaria de México. Psychology/Psicología Reflexão e Crítica, 27(3), 415-423. http://doi. org/10.1590/1678-7153.201427301.

Romero, M. (2016). Pruebas de bondad de ajuste a una distribución normal. Revista Enfermería del Trabajo, 6(3), 105-114.

Sánchez, D., León, S. y Barragán, C. (2016). Correlación de inteligencia emocional con bienestar psicológico y rendimiento académico en alumnos de licenciatura. Investigación en Educación Médica, 4(15), 126-132.

Secretaría de Salud (2013). Programa de acción especifico salud mental 2013-2018. https:// www.gob.mx/cms/uploads/attachment/ file/11918/Salud_Mental.pdf

Sepúlveda, A., Romero, A. y Jaramillo, L. (2012). Estrategias de afrontamiento y su relación con depresión y ansiedad en 
residentes de pediatría en un hospital de tercer nivel. Boletín Médico del Hospital Infantil de México, 69(5), 347-354. http:// www.scielo.org.mx/pdf/bmim/v69n5na5. pdf

Sinha, R. y Jastreboff, A. M. (2013). Stress as a common risk factor for obesity and addiction. Biological Psychiatry, 73(9), 827835. https://doi.org/10.1016/j.biopsych. 2013.01.032

Souza, M. y Cruz, D. (2010). Salud mental y atención psiquiátrica en México. Revista de la facultad de medicina de la UNAM, 53(6), 18-19. http://www.ejournal.unam. mx/rfm/no53-6/RFM053000604.pdf

Tobin D., Holroyd K., Reynolds R. y Wigal J. (1989). The hierarchical factor structure of the Coping Strategies Inventory. Cognitive Therapy Research, 13(4), 343-361.

Valerio, T., Jin, K. y Sexton, K. (2016). Association of stress, general health, and alcohol use with poor sleep quality among U. S. college students. American Journal of Health Education, 47(1), 17-23. http:// doi.org/10.1080/19325037.2015.1111173

Enviado: 19 de febrero de 2020

Revisado: 14 de julio de 2020

Aceptado: 15 de septiembre de 2020 